\title{
Exercício físico e qualidade de vida em idodos: diferentes contextos sociocomportamentais
}

\author{
Exercise and quality of life in the elderly: different social and behavioral \\ contexts
}

\author{
Miguel Camões ${ }^{1 *}$, Fábia Fernandes ${ }^{1}$, Bruno Silva ${ }^{1}$, Tiago Rodrigues ${ }^{1}$, Nuno Costa $^{1}$, Pedro \\ Bezerra1,2
}

ARTIGO ORIGINAL | ORIGINAL ARTICLE

\begin{abstract}
O objetivo deste estudo foi descrever a perceção da qualidade de vida em indivíduos acima dos 70 anos, tendo em conta a participação em programas de exercício físico em contexto comunitário e idosos institucionalizados. Para o efeito foi realizado um estudo transversal onde foram avaliados 250 idosos (74.8\% do sexo feminino) segundo 4 contextos distintos. Grupo $1 \quad(n=148)$ corresponde aos idosos comunitários a praticar exercício (frequência: 2 sessões/semana; duração: 45 minutos); No grupo 2 ( $n=41$ ), incluem-se os idosos que pertencem à comunidade e não fazem exercício; No grupo 3 (centro de dia; $\mathrm{n}=37$ ) e grupo 4 (institucionalizados; $n=24$ ), correspondem aos idosos institucionalizados que não praticam exercício. Para avaliar a perceção da qualidade de vida utilizou-se o questionário SF36. Utilizou-se a regressão linear múltipla para estimar a tendência dos scores por grupo de avaliação. Nos domínios da Função Física, Saúde Mental e Vitalidade, após ajuste para a idade, observou-se uma tendência significativa ( $p$ para a tendência $<0.05$ ) por contexto sócio comportamental, com os valores medianos de qualidade de vida a pertencerem aos idosos envolvidos em programas de exercício. Programas de intervenção com base na prática de exercício físico, mesmo com pouca frequência e duração, relacionaram-se com melhor qualidade de vida em idosos comunitários.
\end{abstract}

Palavras-chave: exercício, qualidade de vida, idosos

ABSTRACT

This study aimed to describe the quality of life of individuals over 70 years, taking into account the participation level in exercise programs, in both community and institutionalized context. Therefore a cross-sectional study was done, with a total of 250 subjects ( $74.8 \%$ female), evaluated according 4 different contexts. Group $1(n=148)$ include community older subjects involved in an exercise program (frequency: 2 sessions/week; time: 45 minutes); Group $2(n=41)$ contain elderly who belong to the community but not involved in regular physical activity; Group 3 (day care center, $n=37$ ) and group 4 (institution, $n=24$ ), aggregate elderly people who attend or are in care centers and did not exercise. To evaluate the perceived quality of life, SF36 questionnaire was performed. Multiple linear regression was done to estimate the trends of the scores according to groups of evaluation. After adjustment for age, significant trends ( $p$ for trend $<0.05$ ) were observed for physical function, mental health and vitality, with the best median results of quality of life belonging to the elderly enrolled in exercise program. Intervention programs based on physical exercise, even with short frequency and duration, are related with better quality of life among community elderly.

Key-words: exercise, quality of life, elderly

Artigo recebido a 01.12.2014; Aceite a 06.07.2015

${ }^{1}$ Instituto Politécnico de Viana do Castelo - Escola Superior de Desporto e Lazer, IPVC-ESDL, Melgaço, Portugal

${ }^{2}$ Centro de Investigação em Desporto, Saúde e Desenvolvimento Humano, CIDESD, Portugal

* Autor correspondente: Escola Superior de Desporto e Lazer - IPVC, Complexo Desportivo e de Lazer

Comendador Rui Solheiro, Monte de Prado, 4960-320 Melgaço, Portugal; E-mail: joaocamoes@esdl.ipvc.pt 


\section{INTRODUÇÃO}

Envelhecer corresponde a um conjunto de processos complexos e naturais que ocorrem desde o nascimento, no entanto as alterações inerentes são mais evidentes numa fase mais avançada da vida (Faria \& Marinho, 2004; Fernandes, 2005). Como fatores do envelhecimento demográfico identificam-se a redução da mortalidade, a redução da fecundidade (número de nascimentos e retardar do projeto de maternidade) e o desenvolvimento e modernização da sociedade (Gil, 2007; Kalache, Veras, \& Ramos, 1987; Pinto, 2006; Rosa, 2012; WHO, 2002). Inerente à redução da mortalidade, encontram-se todo um conjunto de progressos médico/científicos, parcialmente sustentados por evidência observacional e experimental com o ênfase na adoção de comportamentos "saudáveis", sendo estes determinantes na redução do risco de comorbilidades e respetiva mortalidade.

A Organização Mundial de Saúde (OMS) prevê que em 2050 existirão cerca de 2 biliões de pessoas no Mundo com idade superior a 60 anos e que $80 \%$ se encontrará nos países desenvolvidos, como o caso de Portugal (WHO, 2002). Segundo os Censos 2011, avaliações transversais representativas da população Portuguesa, a proporção média de pessoas em Portugal com 65 ou mais anos de idade é de 19,4\%, sendo que na região do Minho-Lima, a prevalência de idosos atinge dimensões ainda mais preocupantes (23,2\%) (I.N.E., 2011). O aumento da longevidade é um grande triunfo para a humanidade. No entanto, torna-se emergente a prevenção e tratamento das patologias crónicas inerentes ao processo de envelhecimento.

Tendo em conta esta nova realidade demográfica, a Organização Mundial de Saúde criou um programa de envelhecimento ativo (Active Ageing) tendo definido algumas linhas de intervenção, ao nível da saúde, participação e segurança (WHO, 2002). Apesar do envelhecimento ser um facto inevitável, o declínio fisiológico varia consideravelmente entre indivíduos (Faina et al., 2008; Faria \& Marinho, 2004), sendo que as perdas inerentes ao envelhecimento natural, podem ser minimizadas com a prática de exercício físico (Bassey, 2002; Matsudo, 2002; Matsudo, Matsudo, \& Neto, 2000; Netto, 2004). Nos dias de hoje, a literatura sustenta de forma consistente, o declínio fisiológico entre os idosos, minimizado pela ação direta do exercício em termos saúde cardiovascular (Vigorito \& Giallauria, 2014), óssea (Granacher, Gollhofer, Hortobágyi, Kressig, \& Muehlbauer, 2013) e também no domínio psicossocial (Franco-Martín, ParraVidales, González-Palau, Bernate-Navarro, \& Solis, 2013).

Uma das linhas de intervenção do programa da OMS para prevenir e reduzir o peso da incapacidade, doenças crónicas e morte prematura nos idosos, é a implementação de programas de atividade física, promovendo consequentemente o envelhecimento ativo (Active Ageing) em detrimento do envelhecimento patológico (Carvalho \& Soares, 2004; Nakagava \& Rabelo, 2007; Phillips \& Davidoff, 2007; Tyndall et al., 2013; WHO, 2002; Zamai, de Moraes, Bankoff, \& Mendes, 2011).

A par do crescimento global do número de idosos, aumentou também o interesse em descrever e compreender como envelhecer de forma mais ativa é um determinante de maior qualidade de vida. Dado que a qualidade de vida se encontra intimamente ligada a um bom desempenho motor, o aumento da aptidão física leva, independentemente da idade, a melhorias significativas no domínio específico do bem-estar físico (Caporicci \& Neto, 2011; Carvalho \& Soares, 2004; Vagetti et al., 2014). Apesar de existir evidência que sustenta esta relação, os dados são pouco consistentes, devido essencialmente às dificuldades de monitorização da população idosa (Carvalho, Fernandes, \& Mota, 2001; Lobo, Santos, Carvalho, \& Mota, 2008; Olivares, Gusi, Prieto, \& HernandezMocholi, 2011; Wanderley et al., 2011).

Com o envelhecimento, a saúde deixa de ser sumariamente medida pela ausência/presença de doença mas sim pela capacidade funcional do idoso. A capacidade funcional está intimamente ligada à manutenção da autonomia e independência, que por sua vez, quando incrementada, permite uma relação clara de 
dependência com a qualidade de vida dos idosos (Kalache et al., 1987; Ramos, 2003).

A qualidade de vida relacionada com a saúde (QdVRS) traduz então a forma como as pessoas percepcionam a sua saúde, traduzindo o bemestar subjetivo do indivíduo em vários domínios, nomeadamente físico (capacidade de realizar tarefas), psicológico (bem-estar emocional e mental) e social (capacidade de se relacionar com as pessoas). Trata-se de um importante indicador da condição atual de saúde, sendo encorajado a sua monitorização em larga escala como forma de estimar a condição real de saúde das populações (Pimentel, 2006).

Apesar de serem escassos os instrumentos de QdVRS desenvolvidos especificamente para a população idosa, a literatura aponta o instrumento genérico 36-item Short Form health survey (SF36) como sendo o mais utilizado (Paixao \& Reichenheim, 2005). Este questionário, criado nos Estados Unidos da América na década de 90 (Ware \& Sherbourne, 1992), foi adaptado e validado para a população portuguesa, seguindo as normas da IQOLA (International Quality of Life Assessment), projeto internacional de adaptação do questionário original em diversos países (Ferreira, 1998; Ferreira, 2000a, 2000b; Ware, Gandek, \& Project, 1998).

Sendo a qualidade de vida um indicador importante da condição atual de saúde nos idosos e tendo em conta os benefícios do exercício, alguns estudos sustentam a comparação de grupos de indivíduos ativos e não ativos envolvidos em programas de intervenção urbano comunitária, Januário, Junior, Liutti, Decker, \& Molari, 2011; Mota, Ribeiro, Carvalho, \& Matos, 2006; Netto, 2004; Orlando, Silva, \& Junior, 2013; Wanderley et al., 2011), outros apenas institucionalizados (Lobo et al., 2008) ou então em centros de convivência (Toscano \& Oliveira, 2009), retratando alterações em alguns indicadores de saúde, estes últimos expostos a um conjunto de fatores, muitas das vezes, fora da realidade populacional.

Em diferentes contextos sócio comportamentais e demográficos, surge a necessidade de descrever a qualidade de vida, tendo em conta os diferentes domínios integrantes da sua definição e recorrendo a instrumentos devidamente validados, em idosos comunitários envolvidos em prática regular de atividade física, expostos a um conjunto de fatores de risco modificáveis, e testar as diferenças para grupos de idosos em comunidade e institucionalizados, não envolvidos em programas de prática estruturada.

\section{MÉTODO}

\section{Participantes}

Estudo observacional descritivo onde foram avaliados 250 idosos, acima dos 70 anos, $(74.8 \%$ do sexo feminino) segundo 4 contextos distintos, pertencentes à região do Alto Minho, concelho de Viana do Castelo, constituindo-se como sendo uma amostra de conveniência. Os idosos foram convidados a participar, tendo por base a divulgação do estudo nos diferentes meios sociais da região (paróquias, centros de saúde e solidariedade social). Após aceitação, os voluntários foram convocados via telefone para proceder às avaliações objetivas nos diferentes centros de solidariedade social e centros de saúde, com maior proximidade das respetivas residências.

Foram constituídos grupos de avaliação observacional, avaliados de forma transversal, abrangendo diferentes contextos sociais e comportamentais. O grupo $1 \quad(n=148)$, corresponde aos idosos comunitários envolvidos num programa de exercício físico. A prática tinha as seguintes dimensões: frequência de 2 sessões/semana; duração de 45 minutos; tipo de atividade de predomínio aeróbio (80\%) e complemento de trabalho anaeróbio/força (20\%). O grupo $2(n=41)$, representa os idosos que pertencem à comunidade e não estão envolvidos em prática regular de atividade física. O grupo $3(n=37)$, corresponde aos idosos que frequentam centros de dia e não fazem exercício físico. O grupo $4(n=24)$, diz respeito aos idosos em instituição e que também não possuem atividades de complemento visando o aumento do dispêndio energético (Tabela 1). 
Tabela 1

Caracterização sociodemográfica e antropométrica dos diferentes grupos de avaliação.

\begin{tabular}{|c|c|c|c|c|c|c|}
\hline & & $\begin{array}{c}\text { Comunidade - } \\
\text { Exercício } \\
(\mathrm{n}=148) \\
\end{array}$ & $\begin{array}{l}\text { Comunidade } \\
\quad(\mathrm{n}=41)\end{array}$ & $\begin{array}{l}\text { Centro de Dia } \\
\quad(n=37)\end{array}$ & $\begin{array}{l}\text { Instituição } \\
(n=24)\end{array}$ & $p$ value \\
\hline & & \multicolumn{5}{|c|}{ Média (dp) } \\
\hline \multirow{2}{*}{\multicolumn{2}{|c|}{ Idade (anos) }} & $74.5(4.1)$ & $77.2(4.8)$ & $82.3(5.9)$ & $81.1(5.2)$ & $<0.001^{*}$ \\
\hline & & \multicolumn{5}{|c|}{$\mathrm{N}(\%)$} \\
\hline Sexo & Feminino & $108(73.0)$ & $30(73.2)$ & $30(81.1)$ & $19(79.2)$ & 0.724 \\
\hline \multirow{3}{*}{ Estado Civil } & Solteiro/a & $9(6.1)$ & $9(22.0)$ & $4(10.8)$ & $7(29.2)$ & \multirow{3}{*}{$0.025^{*}$} \\
\hline & Casado/a & $84(56.8)$ & $16(39.0)$ & $6(16.2)$ & $2(8.3)$ & \\
\hline & Viúvo/a & $55(37.2)$ & $16(39.0)$ & $27(73.0)$ & $15(62.5)$ & \\
\hline & & \multicolumn{5}{|c|}{ Média (dp) } \\
\hline IMC $\left(\mathrm{Kg} / \mathrm{m}^{2}\right)$ & & $29.5(4.1)$ & $29.4(4.3)$ & $34.6(3.1)$ & $30.4(4.9)$ & 0.293 \\
\hline \multirow{4}{*}{ Perímetros $(\mathrm{cm})$} & Braquial & $30.6(3.0)$ & $29.5(3.2)$ & $28.7(3.6)$ & $30.6(3.5)$ & $0.005^{*}$ \\
\hline & Cintura & $104.1(79.9)$ & $96.3(11.4)$ & $97.0(11.0)$ & $99.8(10.1)$ & 0.860 \\
\hline & Coxa & $52.2(8.4)$ & $50.3(4.5)$ & $48.1(5.0)$ & $52.8(14.8)$ & 0.085 \\
\hline & Anca & $106.1(9.6)$ & $106.1(9.6)$ & $105.6(10.8)$ & $108.4(16.5)$ & 0.764 \\
\hline $\begin{array}{l}\text { Distância ao } \\
\text { posto saúde }\end{array}$ & $\mathrm{Km}$ & $3.99(3.86)$ & $2.81(1.76)$ & $1.08(1.46)$ & $1.83(2.01)$ & $<0.001^{*}$ \\
\hline
\end{tabular}

\section{Instrumentos e Procedimentos}

Todos os procedimentos éticos foram assegurados pelo conselho Técnico-Científico do Instituto Politécnico de Viana do Castelo, salvaguardado o anonimato e confidencialidade dos dados dos intervenientes no projeto de investigação, seguindo as recomendações da Declaração de Helsínquia (World Medical Association, 2013).

A informação individual relativa a dados sociodemográficos, comportamentais e antropométricos, foi recolhida por entrevista pessoal com recurso a inquiridores treinados, num único momento de avaliação. As distâncias entre o endereço dos participantes/contexto habitacional e o posto de saúde e/ou hospital de referência, foram obtidas com recurso à ferramenta Google maps ${ }^{\circledR}$.

As medidas antropométricas, peso $(\mathrm{kg})$, estatura $(\mathrm{cm})$ e perímetros (braquial, cintura, anca e coxa; $\mathrm{cm}$ ) foram objetivamente medidas pelos inquiridores, seguindo as normas de International Society for the Advancement of Kinanthropometry (ISAK, 2001). Posteriormente foi calculado o IMC $\left(\mathrm{kg} / \mathrm{m}^{2}\right)$.

Para avaliar a perceção do estado de saúde em idosos foi utilizado o questionário $S F 36_{v 2}$, aplicado sob a forma de entrevista (Lyons \& Perry, 1994), e devidamente validado para aplicação na população Portuguesa (Ferreira, 2000a, 2000b). O SF36 $6_{\mathrm{v} 2}$ é um questionário estruturado, composto por 36 itens que se distribuem em 8 domínios e que permite a avaliação da componente física e mental (Pimentel, 2006). Os domínios são: Função Física (FF), Desempenho Físico (DF), Dor Corporal (DC), Saúde Geral (SG), que constituem a Componente Física (CF), Vitalidade (VT), Função Social (FS), Desempenho Emocional (DE), Saúde Mental (SM), que constituem a Componente Mental (CM) (Ferreira, 1998; Ferreira, 2000a; Ferreira, Ferreira, \& Pereira, 2012; Ferreira \& Santana, 2003; Ware et al., 1998).

O domínio FF pretende medir a limitação para executar tarefas físicas menores tais como vestirse, tarefas intermédias tais como subir escadas, andar a pé, ajoelhar-se ou pegar nas compras de mercearia e tarefas mais extenuantes como correr e levantar pesos. O domínio DF e DE pretendem medir a limitação do sujeito relativamente ao trabalho do dia-a-dia no que diz respeito ao tempo gasto, quantidade de trabalho e a dificuldade a realizar as tarefas. O domínio DC visa representar quer a intensidade da dor mas também a interferência que esta tem no trabalho do sujeito. O domínio SG pretende avaliar a perceção de saúde, a resistência à doença e aparência saudável do sujeito. (Ferreira, 1998; Ferreira, 2000a; Ferreira \& Santana, 2003). O domínio VT inclui níveis de vitalidade e energia para identificar diferenças de bem-estar. A FS pretende aferir a quantidade e qualidade das funções sociais do sujeito mas também em que 
medida o seu estado de saúde interfere com as atividades sociais. Por fim a SM inclui questões sobre ansiedade, depressão, perda de controlo em termos comportamentais e emocionais e o bemestar psicológico (Ferreira, 1998; Ferreira, 2000a; Ferreira \& Santana, 2003).

\section{Análise estatística}

Para a análise dos resultados, utilizou-se o programa informático Statistical Package for Social Sciences (SPPS ${ }^{\circledR}$, versão 19, Chicago, IL). Através da estatística descritiva criaram-se tabelas e gráficos com médias, medianas, desviopadrão, frequências absolutas, de modo a caracterizar a amostra da população em estudo.

Foi testada a normalidade das variáveis recorrendo ao teste de Kolmogorov-Smirnov. Dado as variáveis dependentes não seguirem uma distribuição normal, recorreu-se aos testes não paramétricos. Para comparação de medianas, entre grupos de avaliação, utilizou-se o teste Kruskal-Wallis. Para comparação de proporções, foi usado o teste Qui-Quadrado.

Foi estimada a tendência dos scores dos respectivos domínios do SF36, por grupo de avaliação, através do modelo de regressão linear múltipla, após ajuste para as variáveis que em análise univariada (tabela 1) apresentaram diferenças estatisticamente significativas por grupos de avaliação. Os resíduos do modelo de regressão demonstraram uma homogeneidade de variâncias, permitindo descrever os valores de tendência após ajuste para outras variáveis de interesse. $\mathrm{O}$ valor de significância utilizado foi de $\mathrm{p}<0.05$.

\section{RESULTADOS}

Caraterização dos participantes por contexto sócio comportamental

A proporção de mulheres avaliadas varia entre os $73.0 \%-81.1 \%$ (grupo 1 e grupo 3, respetivamente), não existindo diferenças estatisticamente significativas entre os grupos avaliados $(\mathrm{p}=0.724)$. O grupo 1 apresenta uma média (dp) de idades (anos) significativamente inferior quando comparada com os restantes grupos (74.5 (4.1); 77.2 (4.8); 82.3 (5.9); 81.1 (5.2); grupo $1,2,3,4$, respetivamente). Nas variáveis antropométricas não se verificaram diferenças estatisticamente significativas entre os grupos em análise, com exceção do perímetro braquial (Tabela 1).

No que concerne ao estado marital, este varia entre solteiro/a, casado/a e viúvo/a, sendo pequenas as diferenças na distribuição das proporções por grupos $(\mathrm{p}=0.025)$. Relativamente à distância ao posto de saúde (quilómetros), foram encontradas diferenças estatisticamente significativas $(\mathrm{p}<0.001)$, observando-se que o grupo 1, intervencionado, se encontra mais afastado dos locais em questão (Tabela 1).

\section{Qualidade de vida nos diferentes contextos sócio comportamentais}

Relativamente ao questionário de qualidade de vida (SF36v2), verificaram-se diferenças estatisticamente significativas entre os diferentes contextos sócio comportamentais, nos domínios de saúde geral $(p=0.003)$ e função física $(\mathrm{p}<0.001)$, respetivamente à componente física da qualidade de vida dos idosos avaliados. Distribuições similares foram encontradas nos valores medianos dos domínios da função social $(\mathrm{p}=0.009)$, saúde mental $(\mathrm{p}=0.004)$ e vitalidade $(\mathrm{p}=<0.001)$, respetivos à componente mental (Tabela 2).

No que diz respeito especificamente aos domínios da função física, saúde mental e vitalidade, o grupo 1, envolvido em prática regular de exercício físico, obteve scores finais significativamente superiores quando comparado com o grupo comunitário, centro de dia e instituição, não envolvidos em prática estruturada de atividade física (Tabela 2).

Tendência nos domínios da função física, saúde mental e vitalidade, nos diferentes contextos sociocomportamentais

Nos domínios função física, saúde mental e vitalidade, observa-se uma tendência significativa ( $\mathrm{p}$ para a tendência <0.05) entre os grupos avaliados, após ajuste para a idade, estado civil e distância ao posto de saúde, ilustrada na figura 1 . De salientar que a dispersão observada intra grupo comunidade-exercício, exposto à prática de exercício, permite aferir que os piores resultados registados entre estes idosos, se sobrepõem 
sempre aos valores encontrados nas diferentes componentes que determinam "qualidade de vida", nos idosos institucionalizados. Resultados parcialmente similares foram encontrados nos idosos comunitários intervencionados versus não intervencionados (figura 1).
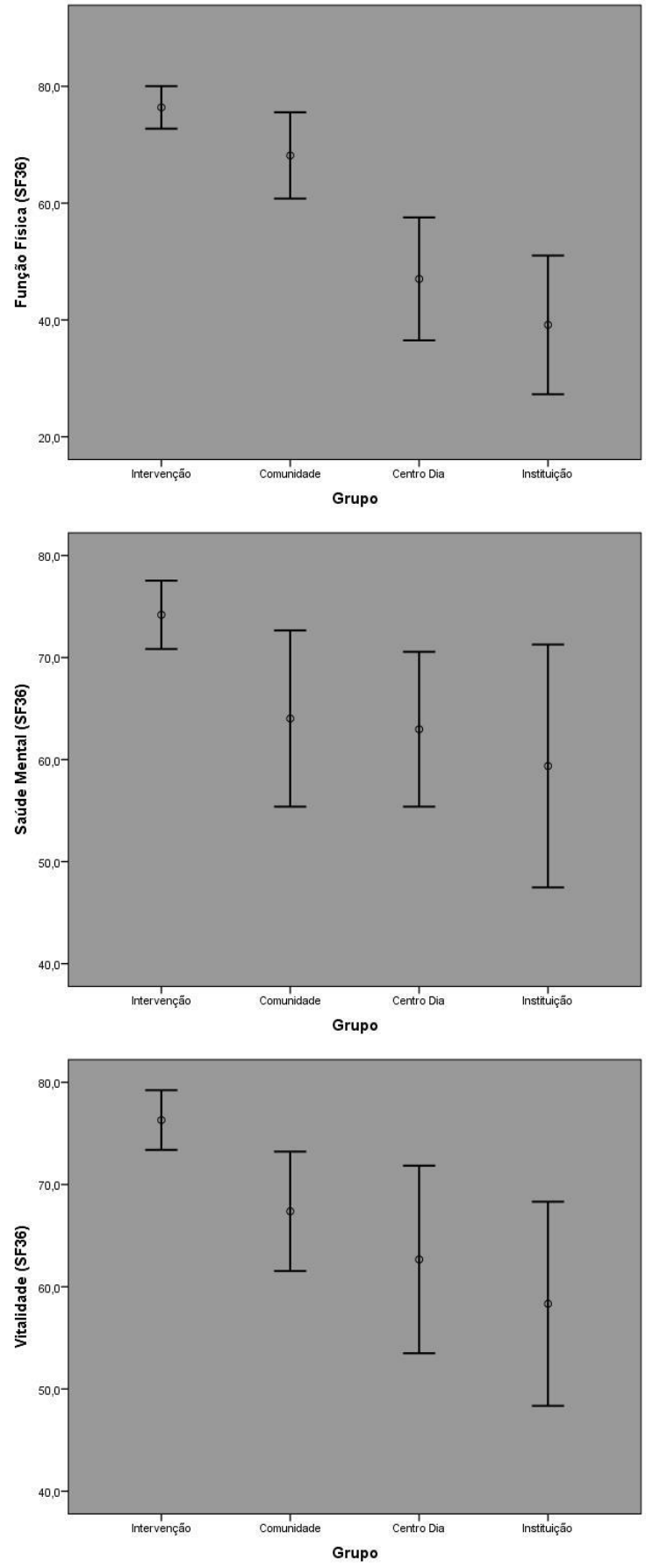

Figura 1. Tendência dos scores medianos nos domínios da função física, saúde mental e vitalidade, por grupos de avaliação.

\section{DISCUSSÃO}

Dos idosos avaliados no presente estudo, aqueles que realizavam prática estruturada de exercício físico, mesmo em contexto comunitário onde se envolvem em ambientes pouco controlados, obtiveram melhores indicadores de qualidade de vida quando comparados com indivíduos comunitários e institucionalizados que não têm atividade física regular. Adicionalmente, observou-se melhores scores no domínio da Função Física, Saúde Mental e Vitalidade no grupo comunitário-exercício e uma tendência decrescente, desses mesmos scores medianos, para os idosos comunitários, centro de dia e institucionalizados, não envolvidos em prática regular.

São vários os estudos a avaliar a qualidade de vida em função dos níveis de atividade física e/ou local de habitação/convívio. O desenho metodológico dos mesmos implica diferentes interpretações e limitam as comparações diretas. Um estudo observacional, com o intuito de avaliar os efeitos da atividade física na autonomia funcional e qualidade de vida dos idosos (idades compreendidas entre 60 e 79 anos), no concelho de Câmara de Lobos (Portugal), tendo utilizado igualmente o SF36 $6_{\mathrm{v} 2}$, dividiu a amostra em praticantes e não praticantes (centro de dia) de atividade física orientada. Observaram-se melhores scores entre os praticantes, nos domínios da FF, DF, DC, VT, FS e DE. Os restantes domínios não apresentaram diferenças estatisticamente significativas entre os grupos de avaliação (Teixeira, 2005). No presente estudo, os indivíduos comunitários intervencionados demonstraram scores consistentemente superiores ao estudo anteriormente referido, mesmo com uma média de idades superior.

Igualmente de base observacional descritiva, mas tendo em conta outro tipo de atividade organizada, existe evidência de uma relação de dependência entre aulas de hidroginástica em mulheres idosas e a sua qualidade de vida (Nakagava \& Rabelo, 2007). Quando comparado com os resultados obtidos para o grupo 1, com prática regular de atividade física, o estudo anterior observou melhores scores médios nos domínios SG, FF, DC e SM. Nos restantes domínios, o nosso estudo revelou melhores resultados, no grupo com prática de exercício. $\mathrm{O}$ tipo de atividade, aulas de hidroginástica, pode ser determinante de variabilidade entre os 
estudos, limitando as comparações diretas quando as variáveis de frequência, intensidade, tipo e tempo de atividade, não são idênticas. $O$ presente estudo acrescenta evidência entre idosos do sexo masculino, quando comparado com o estudo anterior. Apesar das limitações dos estudos observacionais, nomeadamente ao nível da dificuldade em estabelecer uma relação causal, são vários os estudos que sustentam o papel do exercício como determinante de melhor qualidade de vida (Lobo et al., 2008; Toscano \& Oliveira, 2009). $\mathrm{Na}$ análise dos nossos resultados, o ajuste para variáveis de confundimento, como é o caso da idade, estado marital, sexo, distância ao posto de saúde, vem reforçar a relação de dependência entre as variáveis em estudo.

Nos EUA, um estudo experimental avaliou a perda de peso em conjunto com atividade física e qualidade de vida em idosos obesos. Verificou-se que o grupo experimental, exposto a uma dieta e exercício físico (frequência de três vezes por semana; duração de 90min), apresentava melhorias significativas face ao grupo controlo nos domínios FF, DF, DC e VT (Villareal, Banks, Sinacore, Siener, \& Klein, 2006). Tal como era esperado, mesmo em populações específicas, com comorbilidades que se associam de forma significativa com a qualidade de vida, o exercício parece assumir um papel preponderante na forma como os idosos percecionam as diferentes componentes indicadoras de qualidade de vida. $\mathrm{Na}$ nossa amostra, os valores medianos de IMC retratam algum perfil de risco, em todos os grupos sob análise, viabilizando comparações com o estudo anterior. As melhorias significativas entre os indivíduos envolvidos em programas de prática de exercício, vêm em consonância com os resultados obtidos pelo estudo experimental anteriormente referenciado (Villareal et al., 2006). O facto de a evidência advir de um estudo experimental, onde são controladas várias fontes de confundimento, vem aumentar a relevância dos nossos resultados, de base observacional e com maior semelhança ao que acontece em termos populacionais.

Em Portugal, num ensaio clinico com o objetivo de avaliar a qualidade de vida em idosos participantes em programas de atividade física (frequência de duas vezes por semana; duração de 1h) e não participantes, observou-se que os indivíduos do grupo controlo apresentavam pontuação significativamente inferior em todos os domínios do SF36, em comparação com os indivíduos do grupo experimental (Mota et al., 2006). Os valores do SF36, do grupo experimental, foram superiores aos do presente estudo nos domínios FF, DC e SG. Por outro lado, os valores dos domínios SM e DF foram semelhantes aos encontrados entre os idosos avaliados, mesmo com menor volume de exposição ao exercício físico (tempo de atividade) e em contexto comunitário - não experimental. Outros estudos de base experimental, em diferentes contextos socioeconómicos e comportamentais, consolidam a evidência anteriormente descrita (Januário et al., 2011).

Verificam-se diferenças de resultados, entre os estudos, para o grupo mais ativo versus alvo de diversas intervenções. No entanto, estas diferenças na qualidade de vida devem-se aos exercícios aplicados (nível de exposição), ao contexto habitacional (distritos e países diferentes) e ao nível das diferentes características sociodemográficas entre as populações avaliadas.

Ao melhor do nosso conhecimento é escassa a informação observacional, que reúne diferentes contextos habitacionais entre os idosos, a demonstrar diferenças na qualidade de vida dos mesmos. Permite nos assim destacar que, com grande proximidade à realidade populacional, o presente estudo observa melhores indicadores em importantes domínios da qualidade de vida dos idosos comunitários envolvidos em programas de exercício. Dadas as dimensões do exercício no presente estudo (frequência, duração e tipo de atividade), seria simples de replicar as melhorias observadas nos diferentes contextos analisados, sendo mesmo expectável que em ambientes mais controlados (idosos institucionalizados), este tipo de atividades fossem facilmente monitorizadas pelos profissionais de saúde, permitindo um maior impacto na qualidade de vida dos mesmos.

O desenho transversal limita a interpretação ao nível da causalidade. No entanto, o objetivo do estudo não procura uma relação causal, 
viabilizando o poder descritivo da informação. Por outro lado, o tamanho amostral, no grupo de indivíduos institucionalizados, levou a uma maior dispersão dos resultados descritivos, sem impacto na análise devido à utilização das medianas e recurso à estatística não paramétrica. $\mathrm{O}$ ajuste para importantes fatores sociodemográficos, permite acrescentar evidência observacional, em diferentes contextos de sobrevivência. Os dados obtidos através de entrevista pessoal e avaliações objetivas, com recurso a inquiridores treinados, permite assegurar qualidade à evidência encontrada.

\section{CONCLUSÕES}

O grupo de idosos avaliados, inseridos numa zona de Portugal com uma elevada prevalência de indivíduos acima dos 65 anos, apresenta valores de perceção de qualidade de vida positivos (superiores a 50\%), nos diferentes contextos analisados. Como resultado notório destaca-se a tendência decrescente dos scores medianos nos domínios função física, saúde mental e vitalidade, do grupo exposto ao exercício físico regular, para os restantes grupos sob avaliação. De salientar que estas diferenças observam-se mesmo quando as dimensões do exercício são de baixo grau de exigência ao nível da frequência e duração da atividade.

Se em contextos pouco controlados, como é o caso dos idosos comunitários, os resultados parecem demonstrar grande consistência entre os domínios representativos de qualidade de vida, acreditamos que estratégias de prevenção e tratamento com base no exercício físico, devem ser privilegiadas em todos os contextos sócio comportamentais, como forma de manter a autonomia e consequentemente a qualidade de vida, entre a população envelhecida.

\section{Agradecimentos:}

Nada a declarar.

\section{Conflito de Interesses:}

Nada a declarar.

\section{Financiamento:}

Fundos FEDER através do Programa Operacional Fatores de Competitividade - COMPETE e por Fundos Nacionais através da FCT - Fundação para a Ciência e a Tecnologia no âmbito do projeto Estado de Saúde e Atividade Física da População Idosa: PTDC/DTPDES/0209/2012

\section{REFERÊNCIAS}

Bassey, E. (2002). Exercise for the elderly: an update. Age and Ageing, 31(S2), 3-5.

Caporicci, S., \& Neto, M. F. O. (2011). Estudo comparativo de idosos ativos e inativos através da avaliação das atividades da vida diária e medição da qualidade de vida. Motricidade, 7(2), 15-24.

Carvalho, J., \& Soares, J. (2004). Envelhecimento e força muscular - breve revisão. Revista Portuguesa de Ciências do Desporto, 4(3), 79-93.

Carvalho, M. J., Fernandes, R., \& Mota, J. (2001). Efeitos do Exercício Físico na Aptidão Física de Mulheres Idosas. Kinesis, 24, 197-206.

Faina, M., Mirri, G., Manili, U., Cavalazzi, E., Morandini, C., Besi, M., . . . Manno, R. (2008). Physiological and psychological effects of physical exercise on a group of elderly nonexercisers. Medicina Dello Sport, 61(2), 121-138.

Faria, L., \& Marinho, C. (2004). Actividade Física, Saúde e Qualidade de Vida na Terceira Idade. Revista Portuguesa de Psicossomática, 6(1), 93104.

Fernandes, A. (2005). Processos e Estratégias de envelhecimento. Sociologia (15), 223-247.

Ferreira, P. L. (1998). A Medição do Estado de Saúde: Criação da Versão Portuguesa do MOS SF-36: Centro de Estudos e Investigação em Saúde da Universidade de Coimbra, documento de trabalho 2.

Ferreira, P. L. (2000a). Development of the Portuguese version of MOS SF-36. Part I. Cultural and linguistic adaptation. [Criacao da versao portuguesa do MOS SF-36. Parte I--Adaptacao cultural e linguistica.]. Acta medica portuguesa, 13(1-2), 55-66.

Ferreira, P. L. (2000b). Development of the Portuguese version of MOS SF-36. Part II -Validation tests. [Criacao da versao Portuguesa do MOS SF-36. Parte II--Testes de validacao.]. Acta medica portuguesa, 13(3), 119-127.

Ferreira, P. L., \& Santana, P. (2003). Percepção de estado de saúde e de qualidade de vida da população activa: contributo para a definição de normas portuguesas. Revista Portuguesa de Saúde Pública, 21(2), 15-30.

Ferreira, P. L., Ferreira, L. N., \& Pereira, L. N. (2012). Medidas sumário física e mental de estado de saúde para a população portuguesa. Revista Portuguesa de Saúde Pública, 30(2), 163-171.

Franco-Martín, M., Parra-Vidales, E., González-Palau, F., Bernate-Navarro, M., \& Solis, A. (2013). [The influence of physical exercise in the prevention of 
cognitive deterioration in the elderly: a systematic review]. Revista De Neurologia, 56(11), 545-554.

Gil, A. (2007). Envelhecimento Activo: complementaridades e contradições. Sociológico, $17,25-36$.

Granacher, U., Gollhofer, A., Hortobágyi, T., Kressig, R. W., \& Muehlbauer, T. (2013). The importance of trunk muscle strength for balance, functional performance, and fall prevention in seniors: a systematic review. Sports Medicine, 43(7), 627641. https://doi.org/10.1007/s40279-013-00411

I.N.E. (2011). Censos 2011 - Resultados Provisórios. Lisboa: Instituto Nacional de Estatística.

ISAK. (2001). International Standards for Anthropometric Assessment. Underdale: International Society for the Advancement of Kinanthropometry.

Januário, R., Junior, H., Liutti, M., Decker, D., \& Molari, M. (2011). Qualidade de vida em idosos ativos e sedentários. ConScientiae e Saude, 10(1), $112-120$

Kalache, A., Veras, R., \& Ramos, L. (1987). O Envelhecimento da população mundial. Um desafio novo. Revista de Saúde Pública, 21(3), 200-210.

Lobo, A., Santos, P., Carvalho, J., \& Mota, J. (2008). Relationship between intensity of physical activity and health-related quality of life in Portuguese institutionalized elderly. Geriatrics \& Gerontology International, 8(4), 284-290. doi: 10.1111/j.1447-0594.2008.00478.x

Lyons, A., \& Perry, M. (1994). Evidence for the validity of the Short-form 36 Questionnaire (SF36) in an elderly population. Age Ageing, 23(3), 182-184.

Matsudo, S. (2002). Envelhecimento, Atividade Física e Saúde. R. Min. Educ. Fís., 10(1), 195-209.

Matsudo, S., Matsudo, V., \& Neto, T. (2000). Impacto do envelhecimento nas variáveis antropométricas, neuromotoras e metabólicas da aptidão física. Revista Brasileira Ciência e Movimento, 8(4), 2132.

Mota, J., Ribeiro, J., Carvalho, J., \& Matos, M. (2006). Atividade física e qualidade de vida associada à saúde em idosos participantes e não participantes em programas regulares de atividade física. Revista Brasileira Educação Física Esporte, 20(3), 219-225.

Nakagava, B., \& Rabelo, R. (2007). Perfil da Qualidade de Vida de mulheres idosas praticantes de hidroginástica. Revista Digital de Educação Física, 2(1), 1-14.

Netto, F. (2004). Aspectos biológicos e fisiológicos do envelhecimento humano e suas implicações na saúde do idoso. Pensar a Prática, 7, 75-84.

Olivares, P. R., Gusi, N., Prieto, J., \& HernandezMocholi, M. A. (2011). Fitness and health-related quality of life dimensions in community-dwelling middle aged and older adults. Health and Quality of Life Outcomes, 9, 117. https://doi.org/10.1186/1477-7525-9-117
Orlando, M., Silva, M., \& Junior, I. (2013). The influence of the practice of physical activity on the quality of life, muscle strength, balance, and physical ability in the elderly. Revista Brasileira Geriatria Gerontologia, 16(1), 117-126.

Paixao, C. M., Jr., \& Reichenheim, M. E. (2005). A review of functional status evaluation instruments in the elderly. [Uma revisao sobre instrumentos de avaliacao do estado funcional do idoso.]. Cadernos de saude publica, 21(1), 7-19. doi: 10.1590/s0102-311x2005000100002

Phillips, E., \& Davidoff, D. (2007). Normal and Successful Aging: What happens to function as we age. Primary Psychiatry, 1-6.

Pimentel, F. L. (2006). Qualidade de Vida e Oncologia. Coimbra: Almedina.

Pinto, A. (2006). Reflexão sobre o envelhecimento em Portugal. Geriatrics, 2(11), 74-86.

Ramos, L. (2003). Fatores determinantes do envelhecimento saudável em idosos residentes em centro urbano: Projeto Epidoso, São Paulo. Cadernos Saúde Pública, 19(3), 793-798.

Rosa, M. J. V. (2012). O Envelhecimento da Sociedade Portuguesa. Lisboa: Fundação Francisco Manuel dos Santos.

Teixeira, L. (2005). A Actividade Física, Autonomia Funcional e Qualidade de Vida (Dissertação de Mestrado em Ciência do Desporto, na especialidade de Actividade Física para a Terceira Idade). Universidade do Porto, Porto.

Toscano, J., \& Oliveira, A. (2009). Qualidade de Vida em Idosos com Distintos Níveis de Atividade Física. Revista Brasileira Medicina Esporte, 15(3), 169-173.

Tyndall, A. V., Davenport, M. H., Wilson, B. J., Burek, G. M., Arsenault-Lapierre, G., Haley, E., ... Poulin, M. J. (2013). The brain-in-motion study: effect of a 6-month aerobic exercise intervention on cerebrovascular regulation and cognitive function in older adults. BMC Geriatrics, 13, 21. https://doi.org/10.1186/1471-2318-13-21

Vagetti, G., Barbosa Filho, V., Moreira, N., Oliveira, V., Mazzardo, O., \& Campos, W. (2014). Association between physical activity and quality of life in the elderly: a sistematic review, 2000-2012. Revista Brasileira de Psiquiatria, 36(1), 76-88.

Vigorito, C., \& Giallauria, F. (2014). Effects of exercise on cardiovascular performance in the elderly. Frontiers in Physiology, 5. https://doi.org/10.3389/fphys.2014.00051

Villareal, D. T., Banks, M., Sinacore, D. R., Siener, C., \& Klein, S. (2006). Effect of weight loss and exercise on frailty in obese older adults. Archives of Internal Medicine, 166(8), 860-866. https://doi.org/10.1001/archinte.166.8.860

Wanderley, F. A. C., Silva, G., Marques, E., Oliveira, J., Mota, J., \& Carvalho, J. (2011). Associations between objectively assessed physical activity levels and fitness and self-reported health-related quality of life in community-dwelling older adults. Quality of Life Research: An International Journal of Quality of Life Aspects of Treatment, 
Care and Rehabilitation, 20(9), 1371-1378. https://doi.org/10.1007/s11136-011-9875-x

Ware, J. E. J., \& Sherbourne, C. (1992). The MOS 36Item Short-form Health Survey (SF-36): I. Conceptual Framework and Item Selection. Medical Care, 30(6), 473-483.

Ware, J. E., Gandek, B., \& Project, I. (1998). Overview of the SF-36 Health Survey and the International Quality of Life Assessment (IQOLA) Project. Journal of Clinical Epidemiology, 51(11), 903912. doi: $10.1016 / \mathrm{s} 0895-4356(98) 00081$

WHO. (2002). Active Ageing: A policy framework. Spain: OMS.
World Medical Association (2013). WMA Declaration of Helsinki - Ethical Principles for Medical Research Involving Human Subjects. Fortaleza: WMA General Assembly.

Zamai, C. A., Moraes, M. A. A., Bankoff, A. D. P., \& Mendes, R. T. (2011). Atividade Física na Promoção da Saúde e da Qualidade de Vida: Contribuições do Programa Mexa-se UNICAMP. Em G. L. Gutierrez, R. Vilarta, \& R. T. Mendes (Eds.), Políticas Públicas Qualidade de Vida e Atividade Física (1. a ed., Vol. 1, pp. 179-193). São Paulo: IPES EDITORIAL. especificado em contrário e nos conteúdos retirados de outras fontes bibliográficas. 